\title{
Quality of service and fairness for electric vehicle charging as a service
}

\author{
Dominik Danner* and Hermann de Meer \\ From The 10th DACH+ Conference on Energy Informatics \\ Virtual. 13-17 September 2021
}

\section{${ }^{*}$ Correspondence:}

dominik.danner@uni-passau.de University of Passau, Innstraße 41, 94032 Passau, Germany

\begin{abstract}
Due to the increasing battery capacity of electric vehicles, European standard electricity socket-outlets at households are not enough for a full charge cycle overnight. Hence, people tend to install (semi-) fast charging wall-boxes (up to $22 \mathrm{~kW}$ ) which can cause critical peak loads and voltage issues whenever many electric vehicles charge simultaneously in the same area.

This paper proposes a centralized charging capacity allocation mechanism based on queuing systems that takes care of grid limitations and charging requirements of electric vehicles, including legacy charging control protocol restrictions. The proposed allocation mechanism dynamically updates the weights of the charging services in discrete time steps, such that electric vehicles with shorter remaining charging time and higher energy requirement are preferred against others. Furthermore, a set of metrics that determine the service quality for charging as a service is introduced. Among others, these metrics cover the ratio of charged energy to the required energy, the charging power variation during the charging process, as well as whether the upcoming trip is feasible or not. The proposed algorithm outperforms simpler scheduling policies in terms of achieved mean quality of service metric and fairness index in a co-simulation of the IEEE European low voltage grid configured with charging service requirements extracted from a mobility survey.
\end{abstract}

Keywords: Dynamically weighted fair queuing, Electric vehicle charging, Smart grid, Fair charging service allocation, Queuing model, Quality of service

\section{Introduction}

Electric Vehicles (EVs) are seen as one of the key means to reduce the global greenhouse gas emission and air pollution in the transportation sector, especially with the growing use of renewable energy. According to the European Transport Roadmap (European Commission 2011), the European Union encourages the use of EVs to reduce the emission by $80 \%$ to $95 \%$ below 1990 levels by 2050 . The trend towards battery-electrical transportation, the continuously increasing battery storage capacity and driving range of EVs will likely create a high pressure on present power supply infrastructure in the future. Especially the power

(C) The Author(s). 2021 Open Access This article is licensed under a Creative Commons Attribution 4.0 International License which permits use, sharing, adaptation, distribution and reproduction in any medium or format, as long as you give appropriate credit to the original author(s) and the source, provide a link to the Creative Commons licence, and indicate if changes were made. The images or other third party material in this article are included in the article's Creative Commons licence, unless indicated otherwise in a credit line to the material. If material is not included in the article's Creative Commons licence and your intended use is not permitted by statutory regulation or exceeds the permitted use, you will need to obtain permission directly from the copyright holder. To view a copy of this licence, visit http://creativecommons.org/licenses/by/4.0/. 
distribution system may be affected, since many EVs will be charged at home due to convenience and economical reasons (IEA 2020). In order to manage the increasing number of EV charging processes, either the grid must be enhanced to cope with the new peak loads, or an intelligent charging capacity distribution mechanism needs to be established. Because grid expansion is economically and ecologically not always reasonable (Brinkel et al. 2020), intelligent charging control seem to be a promising solution to orchestrate EV charging in the low voltage grid. However, charging control algorithms need to achieve a high Quality of Service (QoS) and Quality of Experience (QoE) in times of grid congestion while ensuring fairness between parallel charging services to retain customers confidence. In this context, electricity (in the form of available grid capacity) can be seen as a limited resource that has to be shared by several end consumers in the power grid. A similar problem exists within the communication networks domain, where several connections share the same physical link with a limited bandwidth. We propose to offer EV customers a charging service that is inspired by computer networking, where only up to a certain bandwidth is provided. The actually received charging current is dynamically adjusted to the grid state and is balanced among charging services to ensure QoS, QoE and fairness.

Solutions in literature that consider (real-time) EV charging allocation (Ardakanian et al. 2013; Ardakanian et al. 2014; Kong et al. 2016; Rudnik et al. 2020; Shi and Liu 2015) aim for proportional fairness on the real-time demand, which is also generally discussed with regard to congestion management (Hekkelman and Poutré 2020) and demand supply matching (Haslak 2020). Schlund et al. (2020) use the laxity of charging processes to enable bidirectional flexibility potential of distributed EV charging processes. Other authors propose price-based solutions (Gan et al. 2011; Hu et al. 2014; Wang et al. 2015). QoS aspects are mainly discussed in combination with charging station sizing (Bayram et al. 2011; Islam et al. 2018; Ul-Haq et al. 2013). However, a few papers investigate QoS and fairness based on other charging parameters in their allocation mechanisms (Frendo et al. 2019; Rezaei et al. 2014; Al Zishan et al. 2020; Zhou et al. 2013; Zhou et al. 2014), but they either ignore the impact on the low voltage grid or do not consider controllability limitations of existing EV communication protocols.

The contribution of this paper can be summarized as follows:

- We first define a set of QoS and QoE metrics in "Requirements for fair charging service allocation" section that consider the ratio of charged energy to the required energy, the continuity of charging rate, the battery State of Charge (SoC) at departure and the ability to reach an upcoming destination.

- Second, we propose an efficient and hierarchically scalable packet queuing allocation mechanism in "Queuing approach for electric vehicle charging" and "Queuing policies" sections that takes the residual charging time and the current SoC into account and ensures fairness between charging services. Our provided model includes not only temporal charging slot allocation (with fixed charging rates), but also distributes the charging capacity during each time slot while respecting charging hardware limitations and control protocol capabilities.

- In "Evaluation" section, our proposed solution is finally evaluated on the IEEE European low voltage test feeder with real user driving profiles extracted from a mobility survey. In contrast to simpler queuing policies, the proposed dynamically 
weighted fair queuing approach achieves both, high QoS results and good fairness indices throughout the whole charging service.

\section{Related work}

There are many papers in literature that deal with coordinated smart charging of EVs targeting the mitigation of power grid issues as their main objective (Alyousef and de Meer 2019; Alyousef et al. 2018; Chung et al. 2014; Lopes et al. 2009; Deilami et al. 2011; Cortés and Martínez 2016; Rivera et al. 2015; Alonso et al. 2014; Kong et al. 2016; Martinenas et al. 2017; Álvarez et al. 2016). However, in this paper we see EV charging is a service to the user, hence the main objective is to make EV drivers happy under the given grid constraints. Therefore, we focus on QoS, QoE and fairness aspects of the charging services.

Nevertheless, there are approaches in literature which try to achieve a certain QoS level of EV charging processes. Some solutions consider public (fast) charging stations as their main focus area and define QoS with respect to the probability that an EV is blocked at a charging station (Bayram et al. 2011; Bayram et al. 2015; Zenginis et al. 2016). In Ul-Haq et al. (2013), all EVs can supply energy back to the charging station and QoS includes also continuity of power supply and overall charging time, whereas the QoS definition in ErolKantarci et al. (2012) only relates to the overall charging time and finishing charging faster implies a higher QoS. Similarly, the QoS in Fan (2012); Haack et al. (2013) is extracted from the charging time, but as binary variable. Only if an EV finishes charging within the required time, it is considered to meet the QoS, no matter what the charging power profile looks like and what final SoC is reached. Our QoS definitions include all metrics from literature and additional QoE metrics that include the circumstances of the charging service to retain customers satisfaction.

None of the above papers consider QoS fairness by design, because most of them are price based or focus only on local charging station sizing (hence only the total QoS for sequential charging processes). In Islam et al. (2016; 2018), photovoltaic and battery sizing are optimized at parking lots for a specific use case of business charging. Their optimization considers QoS as the ratio of charging energy delivered to charging energy demanded. Furthermore, the probabilistic charging model used in Islam et al. (2018) introduces a fairness factor, which influences the charging rate of each single EV based on its SoC. In Ucer et al. (2019) quality of power service is defined such that the voltage drop must be kept stable, but proportional fair charging rates must be provided to all EVs, regardless of their location in the grid. As a result, quality of service to charging EVs is defined on the instantaneous power delivery. Alyousef and de Meer (2019) also focus on the power quality of the grid and implicitly define the QoS in terms of energy delivered to the EV without differentiating the actual energy requirement of the user. Their Transmission Control Protocol (TCP) inspired approach results in a fairer distribution than a purely power quality aware algorithm from Alyousef et al. (2018). Ardakanian et al. (2013; 2014) describe the proportionally fair distribution of available power capacity as an optimization problem that can be solved in a distributed manner via decomposition of its dual problem. The satisfaction of an EV user about a charging process is defined depending on the current charging rate, while the charging service situation such as the $\mathrm{SoC}$ and residual charging duration is neglected. 
The approach in Zhou et al. (2013) shows how available power capacity can be shared fairly using weighted fair queuing scheduling. Their approach is based on packetization of the charging process of EVs, where each packet represents the permission to charge for the next time slot. These packets are queued by the single charging processes to the proposed scheduler, which computes the packet assignment based on their statically determined weight at arrival time. In their solution, demand and supply mismatch defines the available power, whereas we also take a look to the underlying power grid topology. Furthermore, we consider both, the required energy and the remaining time until departure for decision making. Additionally, instead of switching the EVs on and off, EVs receive dynamic charging rates. In Zhou et al. (2014), the same authors compare different scheduling algorithms from the networking context, e.g. round-robin, first come first serve or first depart first serve, for distributing the available power to charging processes. A similar temporal packet-based mechanisms, which uses a probabilistic automaton to limit the transformer utilization by allowing or denying charging requests from distributed EVs, is proposed in Rezaei et al. (2014). According to Chen et al. (2013), pulse charging of EV batteries is not degrading battery lifetime. But switching huge loads of $22 \mathrm{~kW}$ can cause undesired high voltage fluctuations in the grid.

One recent publications on fair charging capacity allocation considers the laxity of a charging process as weights in an optimization problem, which is solved in a decentralized manner (Al Zishan et al. 2020). In order to reduce the impact of users cheating with their departure time, they propose to integrate the reputation of the user into the weights, such that people who estimate their departure time similar exactly obtain the same fair share of power capacity. Despite the fact that (distributed) optimization problems tend to be not very efficient, their output does not integrate actual hardware control capabilities, like limitations of the control protocols or adjustable power limits. Furthermore, fairness is measured on the actual charging power, neglecting other quality of charging service aspects.

In a different application area, Chen et al. (2012) propose a fair power allocation for air conditioners in the smart grid, where the power consumption is indirectly controlled by allocating thermostat settings in each time slot. In this way, ambient temperature and the amount of power required for the same temperature reduction is decoupled and fairness is defined on the QoS level of air conditioning. Similarly in our paper, QoS aspects for a charging service are not necessarily coupled to the actual charging power but measured by charging as a service related metrics.

\section{Methodology}

We first state the requirements for a fair charging resource allocation and define relevant metrics to measure QoS, QoE and fairness of a charging service. Afterwards we describe the proposed queuing architecture and discuss various queuing policies.

\section{Requirements for fair charging service allocation}

The most intuitive notion of fairness is sharing a limited resource proportionally between all participants (EVs), like flow-based fairness in networking (e.g. fairness achieved with TCP). However, some charging processes might require more energy, while the available charging time may be relatively short. Other EVs with longer available charging time require only less energy and, hence, benefit more from proportional fairness. Therefore, 
we will have a look at service-based fairness, where charging services are defined by: (1) the time of arrival $t_{a r r}$ and departure $t_{d e p}$, which together form the available charging time, and (2) the required energy to be charged $E_{\text {req }}>0$. We assume that EV drivers provide their expected departure time and required energy with high confidence, whereby the later can be extracted from the last driven trip. Furthermore, we use a discrete time model with constant time slot size of $\Delta$, where $t_{s t a}$ denotes the first time slot within $\left[t_{a r r}, t_{d e p}\right]$ when the EV is charging and $t_{\text {fin }}$ the time slot when either its battery SoC reaches $100 \%$ or the EV needs to leave at (planned) departure time. Additionally, the charging power profile $P(t)$ is $\geq 0$, because vehicle-to-grid is not considered in this paper. Figure 1 depicts all relevant charging service parameters.

QoS is the measurement of the overall performance of a service and was initially introduced for telecommunication services by ITU in 1994. (ITU 2008). The QoS definition implies that characteristics of a service, which can be measured quantitatively or qualitatively, need to match the users requirements towards the service, and hence involve the user. QoE is defined as "The degree of delight or annoyance of the user of an application or service" (Brunnström et al. 2013). The QoE relates (not necessary linearly) to QoS parameters and additionally integrates the personality and the current state of the user. QoE models are often derived from user surveys, but can also be metrics on the QoS parameters. For example, an EV that can fully recharge its battery during the available charging time receives a high QoS, since the charging service succeeds. If the charging process cannot finish before departure, the QoS is obviously lower. However, if the EV has recharged enough energy to reach the next destination (independent of whether the charging process has finished), the QoE is obviously higher than if an additional charging stop is required during the next trip.

$Q o S_{1}$ The main goal of a charging service is to deliver the required energy $E_{r e q}$ to an EV. Obviously, a finished charging process receives maximal QoS. If the energy target is not met, the QoS degrades proportional to the energy charged. The QoS metric in Eq. 1 can be evaluated at each point in time during the charging process and $\operatorname{QoS}_{1}\left(t_{d e p}\right)$ denotes the final metric score of the charging service.

$$
\mathrm{QoS}_{1}(t)=\frac{E(t)}{E_{\text {req }}}
$$

$\mathrm{QoS}_{2}$ We consider the waiting time of a charging process as second QoS criteria. It follows the logic that a charging process that starts earlier has a higher chance to finish in time. Furthermore, waiting charging processes do not receive any service until the charging process actually starts. Therefore, waiting charging processes receive a lower QoS score, like defined in Eq. 2.

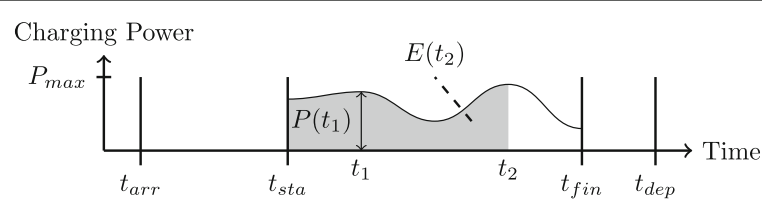

Fig. 1 Overview of a charging service. Note that $t_{\text {fin }}$ can be equal to $t_{d e p}$ 


$$
Q o S_{2}= \begin{cases}1-\frac{t_{\text {sta }}-t_{\text {arr }}}{t_{\text {dep }}-t_{\text {arr }}} & \text { if } \exists P(t)>0, \\ 0 & \text { else }\end{cases}
$$

In case the charging process does not start at all, the charging power profile $(P(t))$ is the identical zero function and hence no $P(t)>0$ exists, which results in $\mathrm{QoS}_{2}=0$. An immediately starting charging process $\left(t_{s t a}=t_{\text {arr }}\right)$ receives highest QoS score.

$\mathrm{QoS}_{3}$ A third QoS criteria is the variation of the charging power $P(t)$ over time. In communication networks we would refer to as packet jitter, which measures the variation of packet delays. For an EV charging service, we focus on the charging power variation between the time slots, where high charging power variation results in bad residual charging time estimation, which in turn reduces the QoS of the feedback towards the user. The respective metric is defined in Eq. 3, where $s(X)$ is the sample standard deviation of a set $X=\left\{x_{1}, \ldots, x_{n}\right\}$.

$$
\mathrm{QoS}_{3}=1-\frac{2 \cdot s(P(t))}{P_{\max }}
$$

The sample standard deviation $s(P(t))$ returns a value from the interval $\left[0, \frac{1}{2} P_{\text {max }}\right]$, because the charging power profile is limit between 0 and $P_{\max }$. Note that for calculating $Q o S_{3}$, values of $P(t)$ are only taken from the interval $t \in\left[t_{s t a}, t_{f i n}\right]$, because only the variation during the actual charging matters.

QoE I $_{1}$ The first QoE metric refers to the battery SoC instead of the actually charged energy of the charging service. Especially, with different battery sizes, but same energy requirements, a user finally does not see the actual energy charged, but only the battery $\mathrm{SoC}$ is displayed in the car. Following this users' recognition, a high $\mathrm{SoC}$ (near to $\mathrm{SoC}_{\text {target }}$ ) corresponds to a high QoE and vice versa. Similar to $Q_{o} S_{1}$, the QoE metric in Eq. 4 can be evaluated at each point in time during a charging process and $Q o E_{1}\left(t_{d e p}\right)$ is the final metric score.

$$
Q o E_{1}(t)=\frac{\operatorname{SoC}(t)}{S o C_{\text {target }}}
$$

$\mathrm{QoE}_{2}$ A second criteria of QoE is whether the EV driver will reach the next destination, which can be expressed as a binary metric like in Eq. 5. In this work, we define the next trip to be feasible if the battery holds enough energy to reach the next destination with a SoC greater than $10 \%$ at arrival. With the remaining SoC it should be possible to reach the next charging facility. This again relates to the users' recognition and range anxiety, which let the driver recharge the battery before running out of energy.

$$
Q o E_{2}= \begin{cases}1 & \text { if next trip is feasible, } \\ 0 & \text { else }\end{cases}
$$

Besides high quality of service and experience scores, fairness among different users matters. A very unfair allocation means that the QoS and QoE metrics differ significantly among the different charging services, whereas very similar metric scores can be considered as fair. Note that in order to analyze fairness separately from the QoS and QoE values, the fairness index must be independent of the metric values. Therefore, we use the fairness index from Hoßfeld et al. (2018) in Eq. 6, where $H$ defines the maximum 
and $L$ defines the minimum possible metric value. The index calculation uses the sample standard deviation $s(S)$ of the metric scores $S=\left\{s_{1}, \ldots, s_{m}\right\}$ of $m$ different charging services.

$$
F(S)=1-\frac{2 \cdot s(S)}{H-L}
$$

Since QoS and QoE metrics from Eq. 1 - (5) are defined within [0,1], the fairness index simplifies to $F(S)=1-2 \cdot s(S)$.

\section{Queuing approach for electric vehicle charging}

In communication networks, multiple information flows are sent simultaneously through the same shared physical link, e.g. using time-division or frequency division multiplexing. For packet switching, often queuing models are used in order to send packets over a network of nodes. Each node holds a queue with packets awaiting transmission to another node. Whenever the communication link is free, a scheduler selects the next packet in the queue, normally based on first-in-first-out policy. In order to establish a certain QoS, other policies can be applied such as earliest deadline first, least laxity first, weighted fair queuing or packet prioritization.

In this paper, each EV is represented by a flow and the EV can request charging currents by scheduling packets to the power grid in discrete time slots, where the packet size $p_{\text {size }}$ equals the EVs' minimal adjustable charging current. This guarantees that EV battery constraints and communication protocol limitations can be considered. For example, if the battery of an EV limits the charging power to $6.9 \mathrm{~kW}$ (10 A on three phases) and the charging current can vary in discrete 3 A steps (1 A per phase) - like defined in IEC 61851-1 - the EV charging service needs to queue 10 packets for each phase. Without loss of generality, we describe the packet allocation for a balanced three-phase power system, hence only a single phase is considered.

The shared network is the underlying power distribution grid whose bandwidth is limited by the available capacities. In order to not overload grid assets, a Scheduling Unit (SU), which contains the queuing logic, is placed at each limiting cable or transformers. Because power distribution grids are typically operated as radial networks, the single SUs span a tree. Each EV requests charging current packets to the nearest connected SU, typically at the supplying cable or transformer. The requested packets pass the network tree towards the root node as depicted in Fig. 2a. Thereby, each SU only forwards as many packets to the next $\mathrm{SU}$ as the local capacity limit allows. Finally, the root node, e.g. responsible for the transformer, assigns its available capacity by returning the packets top-down to the EVs, like depicted in Fig. 2b. Note that voltage violations are treated by a feedback $Q(V)$-controller as described in "Simulation setup" section.

In order to determine the available capacity of a shared link, we propose to measure parts of the distribution grid, and from that infer the available charging capacity of the next time slot using short-term forecasts. The bandwidth calculation can also include an approximation of grid losses, which in turn reduces the actual available charging capacity at the end of the radial network. We assume that applying the allocation algorithm in time slots of one minute is sufficient for charging service allocation and fast enough for reasonable load management in the distribution grid. In addition to the pure network related capacity limitations, the root node can participate in demand response programs 


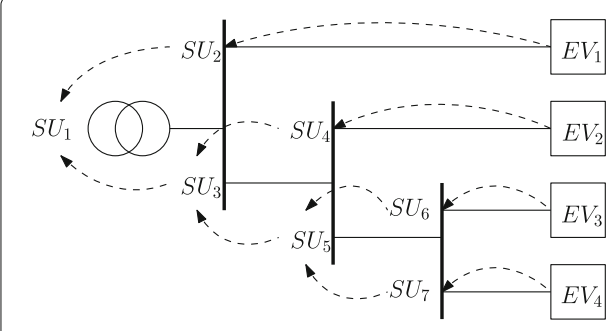

(a) Packet requests.

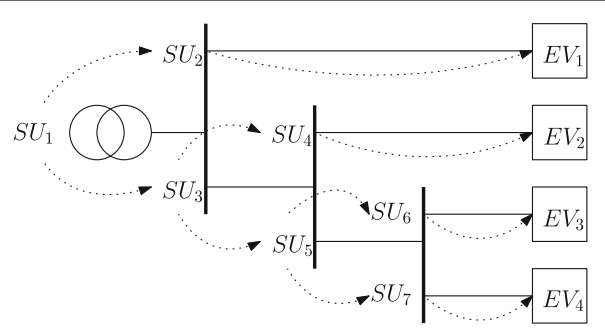

(b) Packet assignments.

Fig. 2 Hierarchical composition of EVs and SUs for the queuing approach

or act as a market agent, which artificially limits the aggregated charging power based on market signals.

The charging capacity $C_{i}(t)$ of an EV $i$ in time slot $t \in \mathbb{N}$ is calculated by $C_{i}(t)=$ $\sum_{p \in A_{i}(t)} p_{\text {size }}$, where $A_{i}(t)$ is the set of packets that is assigned to the EV by the SU. Because EVs that still have packets in the queue might leave before the next iteration, all queues are flushed afterwards. Finally, the allocation algorithm is executed again for the next time slot, starting with packet requests from the EVs.

A reliable and fault-tolerant Information and Communications Technology (ICT) architecture is required for the aforementioned procedure. In case of communication loss between EVs and SUs, the EVs moves to a fail-safe mode while pausing ongoing charging processes to avoid damaging the power grid. Because the SUs are logical units that do not store state information beyond a single time slot, $\mathrm{SU}$ instances can be executed in a cloud environment, which allows fast fault recovery. The communication effort between entities scales linearly with the number of involved EVs and SUs. In each time slot, EVs (leaf nodes in the tree topology) need to send the requested packets along the path of SUs (inner nodes) to the root node, which finally returns them to its origins. Because each EV/SU sends its packet requests only once back and forth in each time slot, the total communication cost can be approximated with $\mathcal{O}(n+m)$ where $n$ is the number of inner nodes and $m$ the number of leaf nodes. Note that this paper does not target privacy nor security issues like packet injection, that may arise with the operation of ICT infrastructure.

\section{Queuing policies}

In the following, we will discuss different simple scheduling policies and, finally, explain the proposed dynamically weighted fair queuing approach.

First Come First Serve (FCFS) The typical implementation of a queue is the first-infirst-out strategy, which means the first element that reaches the queue will be the first element that will be processed. In the case of EV charging, we consider a first come first serve policy. The EV that arrives earlier will be served first with the maximum possible charging current. If there is available capacity left, the EV that arrived next will be served and so forth. Even though considering only the arrival time of EVs for the charging service allocation is simple to realize and secure against malicious user inputs, the flexibility (required energy and residual charging time) of the EV is not considered at all with this scheduling policy. 
Earliest Departure First (EDF) Contrary to the FCFS scheduling, the earliest deadline first policy executes tasks in the order of the nearest deadline. The idea behind this method is to process the more critical tasks first under the assumption that in average each task takes similar execution time. For EV scheduling, earliest deadline first turns into earliest departure first, only considering the departure time of the EV during the scheduling. Similar with FCFS, this policy does not consider the actual required energy and all packets of the same EV are scheduled with the same priority, which results in maximum charging rates for only a few EVs.

Least Laxity First (LLF) The priority of a task is inversely assigned based on its slack time, which is equal to the remaining extra time after job execution until its deadline. Note that the slack time can even be negative in case the job cannot finish in time, which however does not change the execution order. The slack time $s(t)$ at any time $t$ is calculated by $s(t)=d-r(t)-c(t)$, where $d$ is the deadline, $r(t)$ the release time since start and $c(t)$ the residual computation time at time $t$. In EV charging, the departure time is equal to the deadline $\left(d=t_{d e p}\right)$, the time spent within the available charging time equals the release time $\left(r(t)=t-t_{\text {arr }}\right)$ and the required charging time with assumed maximum charging power equals the residual computation time $\left(c(t)=\frac{E_{\text {req }}-E(t)}{P_{\max }}\right)$. Note that for calculating the residual charging time constant current charging with maximum charging power is considered.

Proportional (PROP) Proportional fair scheduling policy guarantees that every participant receives a fair share of a limited resource proportional to its anticipated resource consumption. Proportional fairness is discussed in literature many times with regard to the expected charging power (Ardakanian et al. 2013; Ardakanian et al. 2014; Kong et al. 2016; Shi and Liu 2015; Rudnik et al. 2020), hence we also define proportional allocation based on the charging power requests of the EVs. Note that in our solution proportional fairness is a local property between EVs connected to the same SU. Capacity limitations along the supply grid can prevent global proportional fairness.

Weighted Fair Queuing (WFQ) Packet generalized processor sharing (Demers et al. 1989) can be approximated with weighted fair queuing and is used to share a resource's capacity fairly between flows, while the weight determines the fraction of capacity that each flow receives. Using the WFQ approach in network scheduling, each of $N$ packet flows that passes a shared link is managed by one separate queue $i$ with a specified weight $w_{i} \geq 0$, which is determined by the priority of that flow. Every time a new packet $p$ is received, its virtual finish time is computed by

$$
p_{\text {virtFinish }}=\text { virtStart }_{i}+\frac{p_{\text {size }}}{b_{i}},
$$

where virtStart $i_{i}$ is the last virtFinish time of the same queue $i$ (or the current time if the queue is empty) and $b_{i}$ is the assigned bandwidth to that queue. The bandwidth is calculated by $b_{i}=\frac{w_{i}}{\sum_{j=0}^{N} w_{j}} \cdot R$, using the weights of all single queues $w_{j}$ and the maximum bandwidth $R$ of the shared link. Whenever the scheduler is able to send a packet over the shared link, it selects the queue that contains the packet with the smallest virtFinish time and sends the first packet from that queue. Note that WFQ allocates the resource proportionally fair to the weight of each queue (flow) independent of the packet sizes. 
The pseudo code for requesting packets at a SU is given in Algorithm 1 and the packet assignment is shown in Algorithm 2. In both cases, $Q_{i}$ denotes the packet queue of $\mathrm{EV} / \mathrm{SU} i=1 . . N$, getQueue(p) determines the queue of packet $p$, getNextQueue() returns the queue with the smallest virtFinish time and nextPacket $(Q)$ returns the packet with the smallest virtFinsh time of queue $Q$.

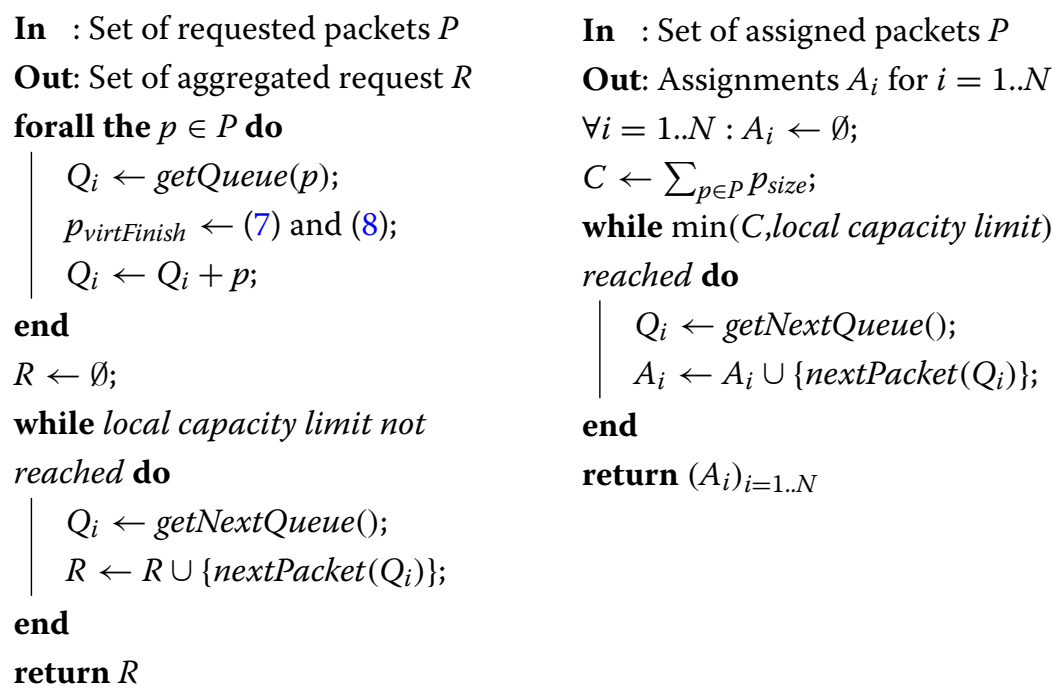

Algorithm 1: Request aggregation. Algorithm 2: Packet assignment.

In networking the packet size $p_{\text {size }}$ denotes the number of bits of the packet and the bandwidth defines how many bits can be transmitted per second (bit/s). For EV charging, the packet size is given by the minimum adjustable charging current. Because the requests are only valid for a discrete time $\Delta$, the actual packet size can be seen as the electrical charge $(A h)$ that needs to be transmitted by the grid. Analogously, the bandwidth is the current carrying capacity of the grid in ampere.

In statically WFQ, the weight of one charging process is once determined at the beginning of the individual charging process when the EV arrives at the charging station. Similar to Zhou et al. (2013), the weight is based on the comparison of the required SoC with the current $\mathrm{SoC}$ of the EV. The weight of EV $i$ is calculated by $w_{i}=\max \left(\mathrm{SoC}_{\text {target }}-\right.$ $\left.S o C\left(t_{\text {arr }}\right), 0\right) \cdot 10+1$. EVs that require a full charge receive weight of 11 and $E V s$ that arrive at home with the required $\mathrm{SoC}$ in the battery obtain a weight of 1 , hence will only charge with minimum priority.

Dynamically Weighted Fair Queuing (DWFQ) By dynamically changing the weights of the flows, WFQ can be utilized to control the QoS for each flow. In contrast to statically weighted fair queuing in Zhou et al. (2013), the dynamically weighted fair queuing approach considers both aspects of the charging service namely, available charging time and required energy. Because for WFQ the weights $w_{i}(t)$ must be $\geq 0$, we cannot use the slack time to dynamically estimate the weight like in LLF policy. Therefore, we divide the remaining charging time $c(t)$ by the remaining time until departure like in Eq. 8. Charging services that theoretically can finish in time receive a weight $\leq 1$, whereas 
others have a weight greater than 1.

$$
w_{i}(t)=\frac{\left(\frac{E_{r e q}-E(t)}{P_{\max }}\right)}{t_{d e p}-t}
$$

\section{Evaluation}

We first explain the underlying experiment setup and assumptions before analyzing the obtained results with regard to QoS and fairness.

\section{Simulation setup}

To evaluate the proposed algorithm in a realistic environment, we extract charging patterns from a mobility survey, state our grid and EV assumptions and define future charging penetration and grid limitation scenarios.

\section{EV charging pattern and battery model}

Nowadays, the driving behavior of people with EV differs to combustion engine drivers, e.g., due to smaller range of the vehicles, limited availability of charging facilities or because EVs are typically used as second car. Nevertheless, we assume that most people will not (like to) change their driving behavior drastically when switching from combustion engine vehicles to electric vehicles in the future. Similar to Danner et al. (2021), we take data from a mobility survey as basis for EV charging behavior, which provides one week of travel behavior in Germany (Eisenmann et al. 2017). This data record contains 1757 surveyed households and more than 2000 individual trips in which the car is the main means of transportation. Each registered trip consists of trip sections with destination, time of departure and arrival, and distance covered.

Because we focus on EV home charging in residential sub-urban area, we filter the survey data to fit to the regional type and aggregate all trip sections such that each trip starts and ends at home. As a result, we obtain the arrival time at home $t_{\text {arr }}$, the departure time from home $t_{d e p}$ and the distance $d$ of the last trip before arriving at home. Because of convenience reasons of the EV driver, we assume that the vehicle will not be charged if the stay between two trips is less than 1 hour, hence the corresponding distance is added to the next trip. In order to get the required energy for the charging service, we assume that all EV drivers want to recharge the consumed energy of the last trip during their stay at home. An exemplary driving pattern that leads to the charging service requirements is depicted in Fig. 3. Assuming a battery storage capacity of $40 \mathrm{kWh}$ and an average energy

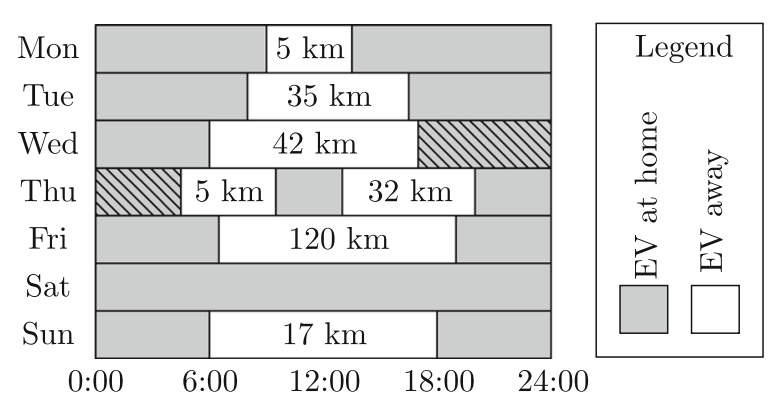

Fig. 3 Exemplary EV charging pattern. During the highlighted charging service, energy for the driven $42 \mathrm{~km}$ need to be charged 
consumption of $17 \mathrm{kWh}$ per $100 \mathrm{~km}$, the required energy of the charging service, which is upper limited by the battery capacity, can be estimated using Eq. 9.

$$
E_{r e q}=\max \left(40 \mathrm{kWh}, d \cdot \frac{17 \mathrm{kWh}}{100 \mathrm{~km}}+E_{m}\right)
$$

In case the battery capacity of an EV is not big enough to cover the whole trip distance, we assume that the driver visits a public charging station during the trip, where only the required additional energy is charged. Hence, with this worst case assumption the EV will arrive at home with an empty battery and requires a full charge cycle. The departure times are assumed to be strict deadlines, meaning a not fully charged EV at departure time that missed to charge energy of $E_{m} \mathrm{kWh}$ requires more energy in the next charging service, respectively.

The number of charging services per EV ranges between 0 and 15 per week. The mean parking duration is approximately $16.6 \mathrm{~h}$ and the mean driving distance is equal to $39.1 \mathrm{~km}$. As can be expected, many commuting EVs reach home between 17:00 and 18:30 and need to leave between 6:30 and 8:00 on the next day. In addition to commuters, the data set also contains $13.2 \%$ shorter charging stops with less than $3 \mathrm{~h}$, which arrive almost with normal distribution around noon.

One of the most common charging models is constant-current-constant-voltage charging, in which increasing battery SoC leads to reduced charging current in the constant voltage phase, also kown as battery saturation phase. This effect is typically observed with charging rates above $50 \mathrm{~kW}$, which are not possible for EV home charging. Therefore, we model the battery charging as constant current load. Furthermore, the charging efficiency is set to $95 \%$ and the minimum adjustable charging current is given by $3 \mathrm{~A}$, similar to the control capabilities in IEC 61851-1.

\section{Power grid and simulation scenarios}

The evaluation is carried out on the simulated IEEE 906 low voltage test feeder. This typical European low voltage grid, shown in Fig. 4, connects 55 households on a three phase system. In our power flow simulation in PyPower within the mosaik co-simulation environment, all households are connected balanced to all three phases. We assume that each

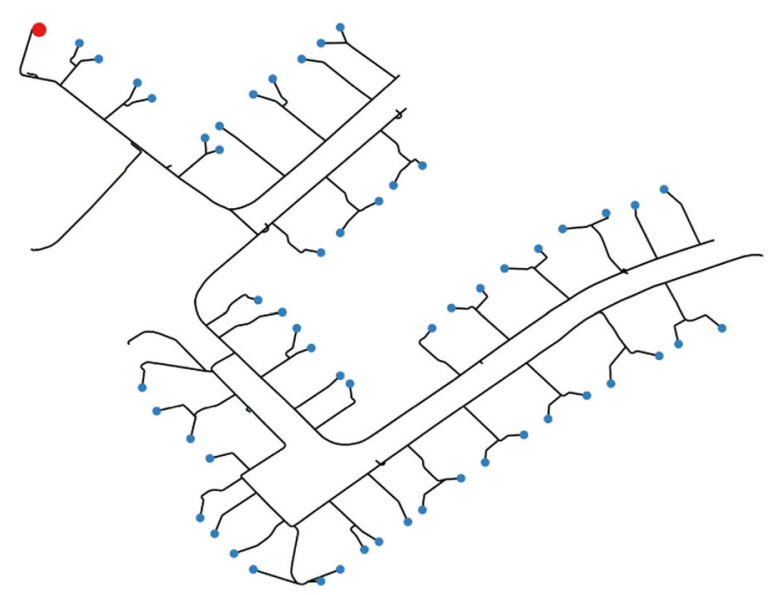

Fig. 4 IEEE 906 low voltage test feeder, with the transformer located in the top left side (red bigger circle) and 55 connected households (blue smaller circles) 

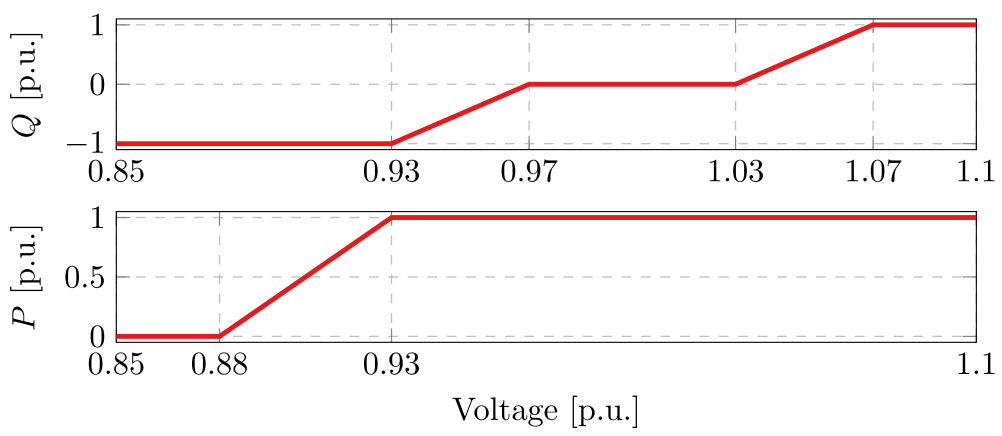

Fig. 5 Voltage controller according to VDE-AR-N 4100

household owns two EVs that can charge in parallel at two 11/22 kW wall-boxes and the aforementioned charging patterns are randomly assigned to the EVs. The very small confidence intervals of 10 independent simulation runs are dropped in "Analysis" section.

In the baseline scenario without EV charging, the low voltage grid has a peak loading of $60.5 \mathrm{kVA}$, which is substantially smaller than the totally maximum installed charging capacity of 2.4 MVA. Nevertheless, uncontrolled charging with $22 \mathrm{~kW}$ and the aforementioned charging patterns results in a peak load of approximately $312 \mathrm{kVA}$ due to the simultaneity factor. Because this would increase the peak load by more than 5 times, which applied to many low voltage grids can cause critical peak loads in the superior power grid infrastructure, and additionally causes voltage issue (details in Table 1), we artificially limit the maximum loading at the transformers' SU to $100 \%$ of the baseline peak load.

The proposed queuing mechanism acts only as load management. Voltage violations are counteracted by $Q(V)$ and $P(V)$ droop curves like in Fig. 5. The decentralized voltage controller changes the reactive power behavior of the rectifier and in critical situations even reduces the real power demand of the EV. Note that in our simulation the power factor is configured to be always greater than 0.9 to avoid losses and keep the reactive power ratio in the low voltage grid in a reasonable range. Therefore, the real power demand is slightly reduced between 0.93 and 0.97 p.u. (1.03 and 1.07 p.u. respectively) in order to not exceed the allocated current capacity. Furthermore, the reactive power decreases with the real power demand below 0.93 p.u. in order to stay with the defined minimum power factor. Although this voltage controller might reduce the actually obtained charging capacity for EVs at critical locations in the grid, LLF and DWFQ restore fairness between different charging services by dynamically recalculating the weights. In order to avoid osculations, we apply a first-order lag filter to the control signal changes.

$$
\begin{aligned}
& P(t)=k \cdot \hat{P}(t)+(1-k) \cdot P(t-1) \\
& Q(t)=k \cdot \hat{Q}(t)+(1-k) \cdot Q(t-1)
\end{aligned}
$$

$\hat{P}(t)$ and $\hat{Q}(t)$ are the target signal value from the voltage controller limited by the assigned charging current from the queuing mechanism. The factor $k$ must be configured to avoid oscillation, but still reach the target signal value within desired time. Our co-simulation steps with $\Delta$ equal to $1 \mathrm{~min}$ and the target value is nearly reached after 5 steps using $k=1-\frac{1}{e} \approx 0.632$ to provide a fast enough reaction. 


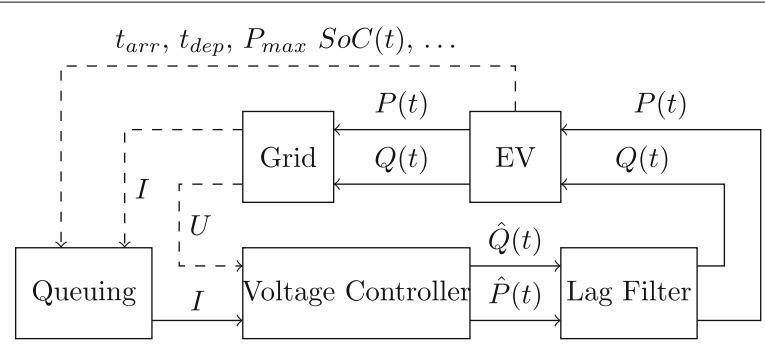

Fig. 6 Control flow in the co-simulation, where dashed lines are time delayed

The control flow of the co-simulation, visualized in Fig. 6, first executes the queuing mechanisms to obtain the assigned charging current $I$. Secondly, using the locally measured voltage $U$ the voltage controller calculates the real and reactive power values of the charging service $\hat{P}(t)$ and $\hat{Q}(t)$. Next, the first-order lag filter is applied before the parameters are sent to the EV model, which passes the values to the power flow simulator. The calculated $\mathrm{SoC}$ of the EV, available current capacity and node voltage form the grid close the control loop, which is executed every $1 \mathrm{~min}$.

\section{Analysis}

All the following results are obtained from a 7-day week simulation. During this simulated week, the individual EVs require between 1 and 13 charging services, in average 5.06. The mean energy demand of a charging service is $6.68 \mathrm{kWh}$, which is approximately $16.7 \%$ of the assumed battery capacity. The total energy demand of all 557 charging services is $13.2 \%$ greater than the total demand of the households.

\section{QoS, QoE and fairness index of the different queuing policies}

First, we analyze the obtained metric values for the quality of service and experience of the different queuing policies, where Fig. 7 shows box plots of all 557 charging services. All policies achieve high $Q o S_{1}, Q o E_{1}$ and $Q o E_{2}$ values that are close to one for most charging services, however the number and variation of outliers varies significantly among the queuing policies. In the charging service metric $Q o S_{1}$, FCFS, PROP and WFQ have slightly lower averages due to many outliers, and there are even some charging services that do not receive any service at all. That is the case, when charging services are blocked by other charging service that actually provide more flexibility to be moved to a later time. In all three metrics ( $Q o S_{1}, Q o E_{1}$ and $\left.Q o E_{2}\right)$, EDF and DWFQ achieve maximum quality with the exception for $Q o E_{2}$ where some charging service are rated with poor QoE because a fully charged battery is not enough to reach the next destination. The in-cooperation of departure times plays an important role for charging service allocation due to the better performance of EDF, LLF and DWFQ in these three metrics.

The two metrics $\mathrm{QoS}_{2}$ and $\mathrm{QoS}_{3}$, which target QoS during the charging time (starting time and power variation), show a different picture. For both, the average value of the first three policies (FCFS, EDF and LLF) is noticeable lower than with the last three policies. This is due to the fact that the later three also enable variable charging currents, whereas the first three policies operate as purely time division multiplexing. Blocked by other services the start times of charging services are delayed, which affects $\mathrm{QoS}_{2}$, and charging interrupts of newly arriving EVs increase the variation in charging power, which is reflected in $\mathrm{QoS}_{3}$. 


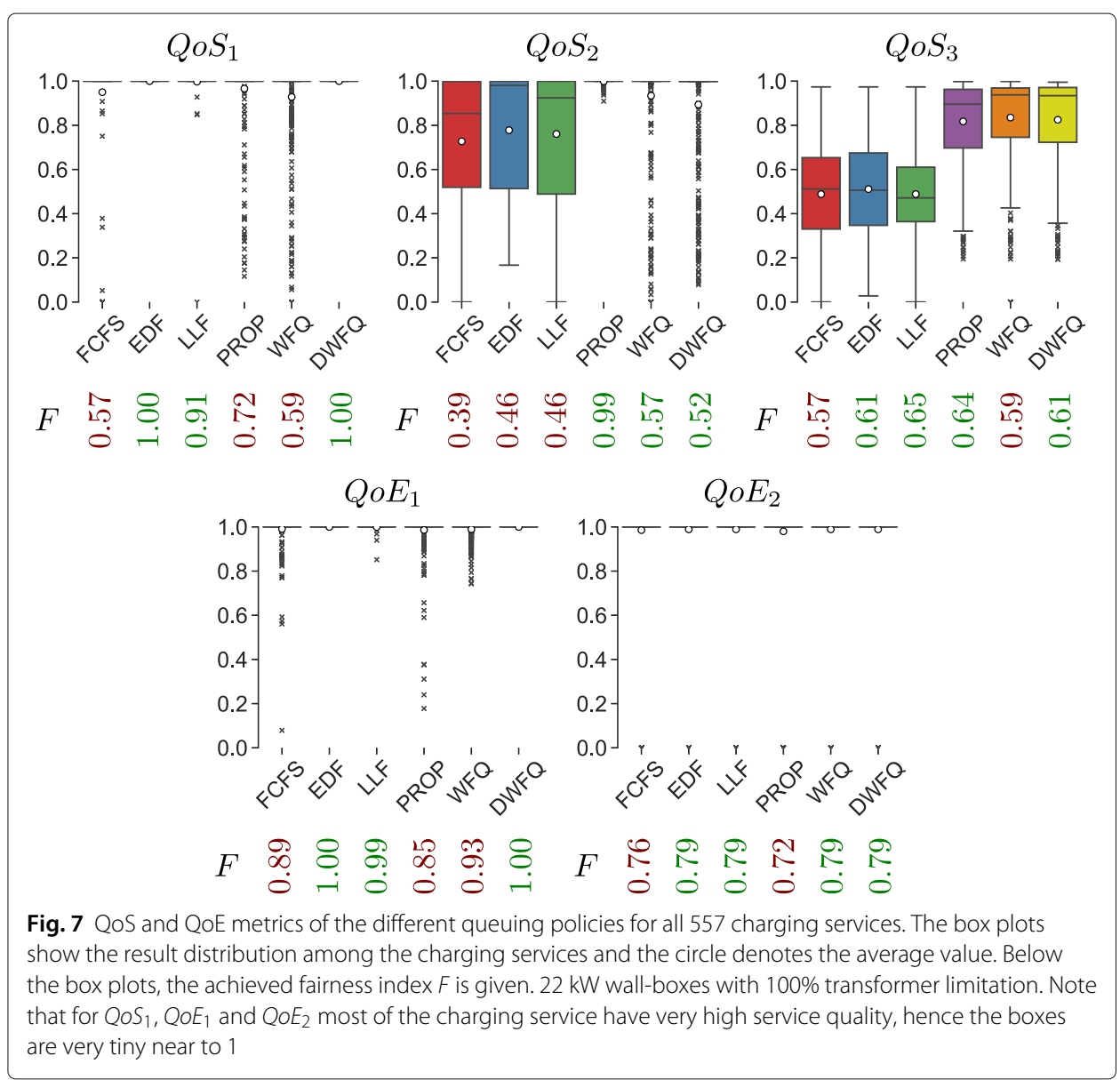

Below the box plots in Fig. 7, the fairness index $F$ is depicted for each metric and policy. As can be seen, only the proposed DWFQ policy is among the best three for each metric, whereas EDF and LLF achieve a high fairness index for most metrics except $\mathrm{QoS}_{2}$. Again, this tracks back to the time division behavior of both policies. Figure 8 compares the mean quality of service and experience obtained with the fairness index. The spider plots clearly show that PROP, WFQ and DWFQ outperform the other three policies in terms of mean

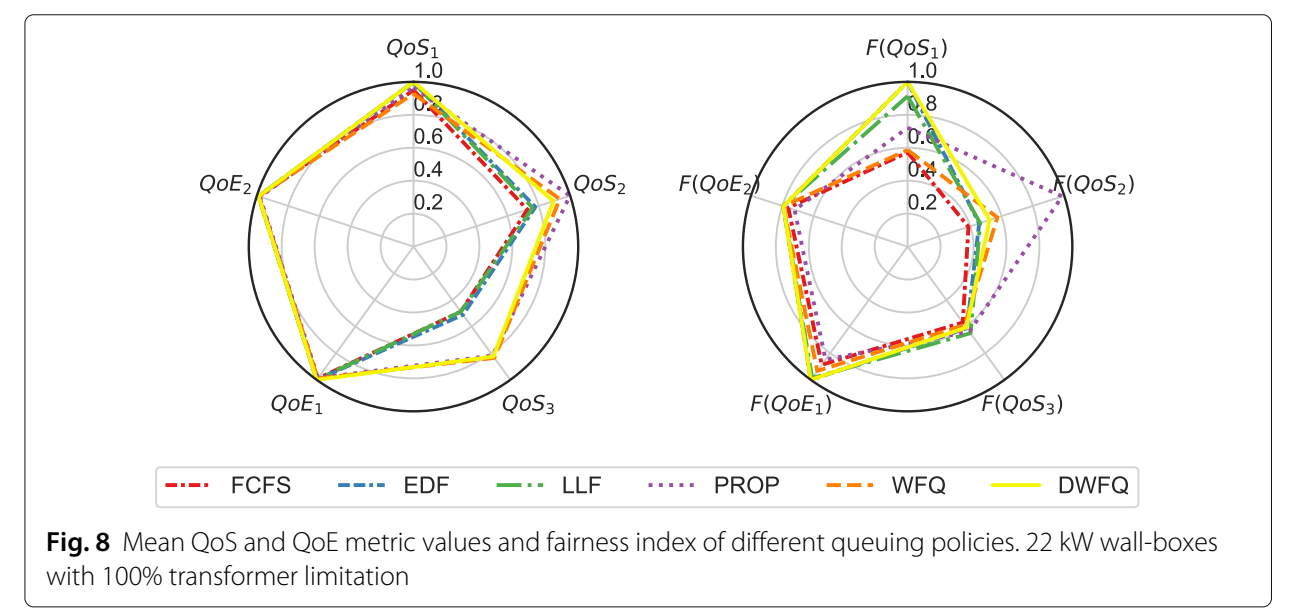


value of all quality metrics. Additionally, DWFQ achieves good fairness indices in most metrics, which makes it a good candidate for fair charging service allocation.

Note that similar results with less differences between the queuing policies are obtained with a transformer limitation of $120 \%$ and $140 \%$ or $11 \mathrm{~kW}$ wall-boxes, respectively. It can even be expected that with unlimited transformer (and cable) limitation, all policies work similar, since all charging requests can directly be served. Nevertheless, the best queuing policy should be chosen in order to ensure high quality of charging service and fairness even with highly limited scenarios.

\section{QoS, QoE and fairness index during the charging service}

As already identified in the last section, the quality of service and experience during the charging services differs quite a lot between the queuing policies. Figure 9 depicts the evolution of the $Q o E_{1}$ mean value and fairness index during charging. Note that for the $\mathrm{x}$-axis all charging services are normalized to the range between arrival $t_{\text {arr }}$ and departure time $t_{d e p}$. This makes them comparable on the same time scale, even though the charging services have different duration and do not take place at the same time. From Fig. 9a it can be seen that the mean $Q o E_{1}$ value of all policies evolves quite similarly during charging. Compared to the other policies, FCFS has a slightly lower value during the first half of the charging services, because newly arrived EVs are blocked until all previous charging services are fully served. Only EDF, LLF and DWFQ finally reach the maximum at departure time. Because EDF and LLF schedule only maximum charging power to the most critical

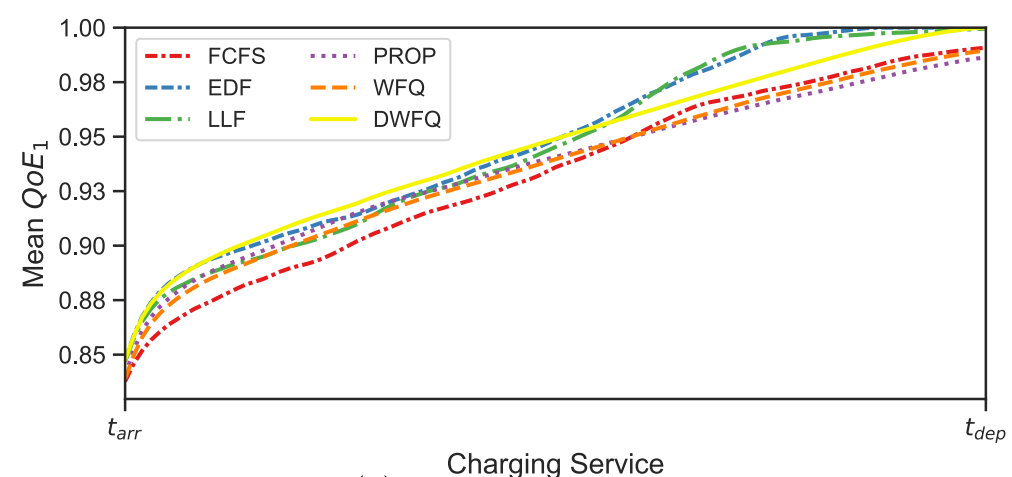

(a) Mean $Q \circ E_{1}$ value

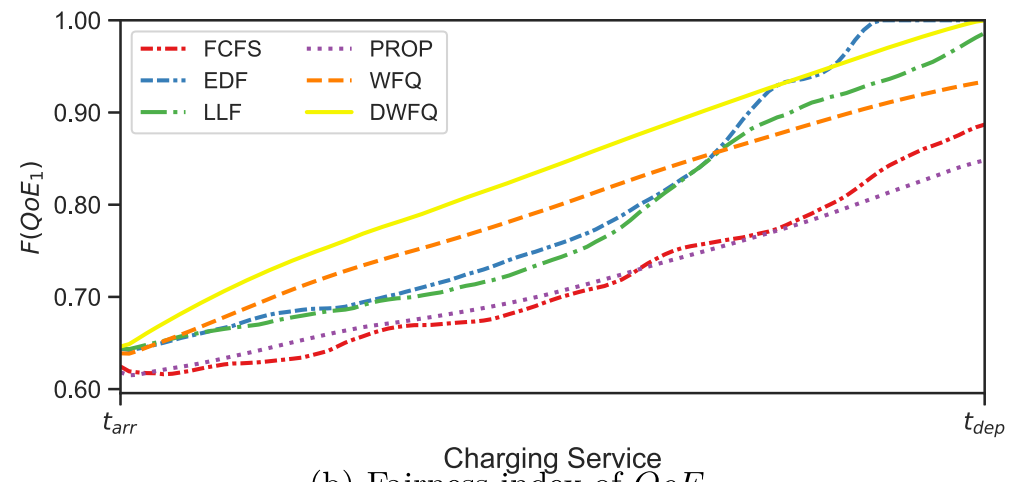

(b) Fairness index of $Q o E_{1}$

Fig. 9 Mean $Q o E_{1}$ metric value and fairness index during the charging services using different queuing policies. All charging processes are normalized to the range $\left[t_{\text {arr }}, t_{\text {dep }}\right]$. $22 \mathrm{~kW}$ wall-boxes with $100 \%$ transformer limitation 


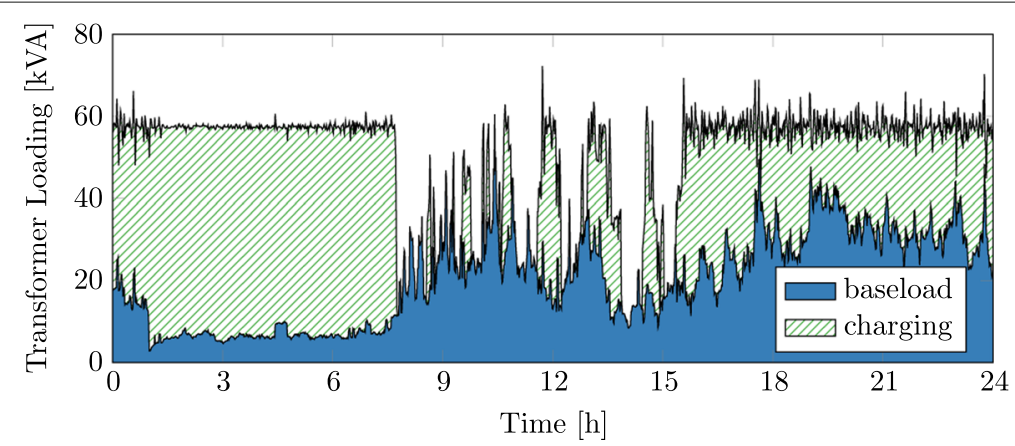

Fig. 10 Loading at the transformer during one day, limited to 100\% of the baseline peak load. EV charging with $22 \mathrm{~kW}$ using the DWFQ policy

charging processes with regard to time and remaining available charging time, both reach the maximum metric value earlier than DWFQ. In contrast, DWFQ focuses on a fair allocation throughout the whole charging process, which results in a higher mean quality metric at the first half of the charging service. Despite the fact that DWFQ has a slightly lower mean $Q o E_{1}$ value at the last third of the charging service, most of the time this policy dominates the fairness index shown in Fig. $9 \mathrm{~b}$ except at the very end. With a higher fairness index during the first half of the charging service, EVs are served more fairly in case they need to leave earlier than the planned departure time. Furthermore, it can be expected that DWFQ (and also WFQ) are more robust against malicious user inputs (e.g. incorrect departure times), because opposite to EDF all charging services always obtain a fair portion of the available charging capacity according to their weight.

\section{Impact of the charging service on the low voltage grid}

Regardless of which queuing policy is used to distribute the available charging capacity, the load at the transformer is smoothly limited to the configured threshold, except for a few short violations as shown in Fig. 10. Table 1 summarizes the achieved mean and minimum SoC at departure time ( $\left.Q o E_{1}\right)$ of the different queuing policies and also provides grid statistics extracted from the power flow simulation. We take the minimum of the 10minutes average voltage values (as defined in EN 50160) at all buses as an indicator for the voltage impacts of the different queuing policies. The grid losses are calculated by comparing the charged energy of all charging services with the additional energy that passes

Table 1 Charging statistics and impact on the low voltage grid of the different queuing policies

\begin{tabular}{lllll}
\hline Policy & Mean SoC $\left(Q o E_{1}\right)$ & Min SoC $\left(Q o E_{1}\right)$ & Min $U_{10 \min }^{\text {avg }}$ & Grid losses \\
\hline FCFS & $99.76 \%$ & $77.09 \%$ & $217.91 \mathrm{~V}$ & $7.35 \%$ \\
EDF & $\mathbf{1 0 0} \%$ & $\mathbf{1 0 0} \%$ & $216.68 \mathrm{~V}$ & $7.44 \%$ \\
LLF & $\mathbf{1 0 0 \%}$ & $\mathbf{1 0 0} \%$ & $216.35 \mathrm{~V}$ & $7.42 \%$ \\
PROP & $99.78 \%$ & $69.44 \%$ & $\mathbf{2 2 0 . 9 4} \mathbf{~ V}$ & $\mathbf{6 . 5 4 \%}$ \\
WFQ & $99.76 \%$ & $81.99 \%$ & $\mathbf{2 2 0 . 1 9} \mathbf{~ V}$ & $\mathbf{6 . 5 1 \%}$ \\
DWFQ & $\mathbf{1 0 0 \%}$ & $\mathbf{1 0 0} \%$ & $\mathbf{2 1 9 . 1 4} \mathbf{~ V}$ & $\mathbf{6 . 5 8 \%}$ \\
Baseline & - & - & $231.55 \mathrm{~V}$ & $0 \%$ \\
uncontrolled & $100 \%$ & $100 \%$ & $176.69 \mathrm{~V}$ & $12.79 \%$ \\
U-control & $100 \%$ & $100 \%$ & $203.67 \mathrm{~V}$ & $11.88 \%$ \\
\hline
\end{tabular}

$22 \mathrm{~kW}$ wall-boxes with $120 \%$ transformer limitation. $U_{10 \mathrm{~min}}^{\mathrm{avg}}$ is the 10 -minute average voltage value according to EN 50160. Top three scores are marked boldface 
the transformer. All queuing policies reach an acceptable voltage level, but the three policies with variable charging rates improve the voltage level by more than $2 \mathrm{~V}$ compared to the other policies. Similarly, grid losses are reduced by approximately $1 \%$ (approximately $37 \mathrm{kWh}$ ). This is due to the fact that the total charging capacity is shared among more charging services, with each receiving a smaller share, thereby reducing the voltage drop and grid losses. Note that this study does not consider that EV charging equipment might have lower efficiency when not utilized with the rated power. Values from the baseline scenario without charging (Baseline), uncontrolled charging (uncontrolled) and only using the aforementioned local voltage controller ( $U$-control) are given in Table 1 for comparison.

\section{Conclusion and future work}

This paper presented a set of QoS and QoE metrics that can be used to evaluate EV charging services. Among others, the charged energy, charging power variations and whether the next destination can be reached with the charged energy are considered. Secondly, we have proposed a hierarchically scalable charging allocation mechanism that uses queuing systems and can apply various queuing policies, e.g. first come first served, earliest departure first or least laxity first. The proposed charging solution can capture charging restrictions coming from the battery or legacy communication protocols between the wall-boxes and the EV. Three of the analyzed queuing policies provide decent QoS and QoE in all five metrics while achieving a better overall fairness compared to the other policies in our co-simulation. Due to the variable charging rates and the dynamic recalculation of the weight (using the remaining available charging time and the remaining required energy), the proposed DWFQ is among the best and additionally has only small negative effect on voltage levels and grid losses. Finally, all charging services in our simulation - extracted from a mobility survey - are sufficiently served with a transformer power limitation of $100 \%$ of its normal baseline load without EV charging. Therefore, we have demonstrated that with an advanced charging service allocation the demand of high EV penetration can be met with the same peak load as the baseline scenario without EV, however QoS and fairness highly depend on the chosen allocation policy.

In the future, we want to perform a sensitivity analysis on how malicious user inputs impact the QoS and fairness and evaluate how incentive mechanisms can improve the impact of wrong inputs. Additionally, we plan to compare the discussed hierarchical charging mechanism with a fully decentralized probabilistic allocation protocol that uses multiple access control mechanisms from the networking domain.

\footnotetext{
About this supplement

This article has been published as part of Energy Informatics Volume 4 Supplement 3, 2021: Proceedings of the 10th DACH+ Conference on Energy Informatics. The full contents of the supplement are available online at https://energyinformatics. springeropen.com/articles/supplements/volume-4-supplement-3.
}

Authors' contributions

DD contributed the idea, service quality metrics, algorithms as well as the evaluation setup and analysis. He also wrote the first draft of the paper. HdM provided research direction, supervision, and helped to write the final version of the paper. Both authors read and approved the final manuscript. 
Availability of data and materials

The co-simulation environment with all connected simulators and results are available from the corresponding author on reasonable request.

\section{Declarations}

\section{Competing interests}

The authors declare that they have no competing interests.

\section{Published: 13 September 2021}

\section{References}

Álvarez JN, Knezović K, Marinelli M (2016) Analysis and comparison of voltage dependent charging strategies for single-phase electric vehicles in an unbalanced danish distribution grid. In: 2016 51st International Universities Power Engineering Conference (UPEC)

Al Zishan A, Moghimi Haji M, Ardakanian O (2020) Reputation-based fair power allocation to plug-in electric vehicles in the smart grid. In: 2020 ACM/IEEE 11th International Conference on Cyber-Physical Systems (ICCPS). IEEE, Sydney. pp 63-74

Alonso M, Amaris H, Germain J, Galan J (2014) Optimal charging scheduling of electric vehicles in smart grids by heuristic algorithms. Energies 7(4):2449-2475

Alyousef A, Danner D, Kupzog F, de Meer H (2018) Enhancing power quality in electrical distribution systems using a smart charging architecture. Energy Inf 1(S1):28

Alyousef A, de Meer H (2019) Design of a TCP-like Smart Charging Controller for Power Quality in Electrical Distribution Systems. In: Proceedings of the Tenth ACM International Conference on Future Energy Systems (e-Energy '19). Association for Computing Machinery, Phoenix. pp 128-138

Ardakanian O, Keshav S, Rosenberg C (2014) Real-Time Distributed Control for Smart Electric Vehicle Chargers: From a Static to a Dynamic Study. IEEE Trans Smart Grid 5(5):2295-2305

Ardakanian O, Rosenberg C, Keshav S (2013) Distributed control of electric vehicle charging. In: Proceedings of the Fourth International Conference on Future Energy Systems (e-Energy '13). Association for Computing Machinery, Berkeley. pp 101-112

Bayram IS, Michailidis G, Devetsikiotis M, Bhattacharya S, Chakrabortty A, Granelli F (2011) Local energy storage sizing in plug-in hybrid electric vehicle charging stations under blocking probability constraints. In: 2011 IEEE International Conference on Smart Grid Communications (SmartGridComm). IEEE, Brussels. pp 78-83

Bayram IS, Tajer A, Abdallah M, Qaraqe K (2015) Capacity planning frameworks for electric vehicle charging stations with multiclass customers. IEEE Trans Smart Grid 6(4):1934-1943

Brinkel NBG, Schram WL, AISkaif TA, Lampropoulos I, van Sark WGJHM (2020) Should we reinforce the grid? cost and emission optimization of electric vehicle charging under different transformer limits. Appl Energy 276:115285

Brunnström K, Beker SA, De Moor K, Dooms A, Egger S, Garcia M-N, Hossfeld T, Jumisko-Pyykkö S, Keimel C, Larabi M-C, Lawlor B, Le Callet P, Möller S, Pereira F, Pereira M, Perkis A, Pibernik J, Pinheiro A, Raake A, Reichl P, Reiter U, Schatz R, Schelkens P, Skorin-Kapov L, Strohmeier D, Timmerer C, Varela M, Wechsung I, You J, Zgank A (2013) Qualinet White Paper on Definitions of Quality of Experience. https://hal.archives-ouvertes.fr/hal-00977812. Accessed 08 Mar 2021

Chen YW, Chen X, Maxemchuk N (2012) The fair allocation of power to air conditioners on a smart grid. IEEE Trans Smart Grid 3(4):2188-2195

Chen LR, Wu SL, Shieh DT, Chen TR (2013) Sinusoidal-ripple-current charging strategy and optimal charging frequency study for li-ion batteries. IEEE Trans Ind Electron 60(1):88-97

Chung C, Chynoweth J, Chu C, Gadh R (2014) Master-slave control scheme in electric vehicle smart charging infrastructure. Sci World J 2014:14

Cortés A, Martínez S (2016) A hierarchical algorithm for optimal plug-in electric vehicle charging with usage constraints. Automatica 68:119-131

Danner D, Seidemann J, Lechl M, de Meer H (2021) Flexibility disaggregation under forecast conditions. In: Proceedings of the Twelfth ACM International Conference on Future Energy Systems (e-Energy '21). Association for Computing Machinery, New York. pp 27-38

Deilami S, Masoum AS, Moses PS, Masoum MAS (2011) Real-Time Coordination of Plug-In Electric Vehicle Charging in Smart Grids to Minimize Power Losses and Improve Voltage Profile. IEEE Trans Smart Grid 2(3):456-467

Demers A, Keshav S, Shenker S (1989) Analysis and simulation of a fair queueing algorithm. SIGCOMM Comput Commun Rev 19(4):1-12

Eisenmann C, Chlond IB, Hilgert T, von Behren S, Vortisch IP (2017) Deutsches mobilitätspanel (mop)-wissenschaftliche begleitung und auswertungen bericht 2016/2017: Alltagsmobilität und fahrleistung. Technical report, KIT

Erol-Kantarci M, Sarker JH, Mouftah HT (2012) Quality of service in plug-in electric vehicle charging infrastructure. In: 2012 IEEE International Electric Vehicle Conference. IEEE, Greenville. pp 1-5

European Commission (2011) White Paper on transport: Roadmap to a Single European Transport Area - towards a competitive and resource efficient transport system. https://eur-lex.europa.eu/legal-content/EN/TXT/PDF/?uri= CELEX:52011DC0144\&from=EN. Accessed 08 Mar 2021

Fan Z (2012) A distributed demand response algorithm and its application to phev charging in smart grids. IEEE Trans Smart Grid 3(3):1280-1290

Frendo O, Gaertner N, Stuckenschmidt H (2019) Real-time smart charging based on precomputed schedules. IEEE Trans Smart Grid 10(6):6921-6932

Gan L, Topcu U, Low S (2011) Optimal decentralized protocol for electric vehicle charging. In: 2011 50th IEEE Conference on Decision and Control and European Control Conference. IEEE, Orlando. pp 5798-5804

Haack J, Akyol B, Tenney N, Carpenter B, Pratt R, Carroll T (2013) Volttron ${ }^{\text {TM: }}$ : An agent platform for integrating electric vehicles and smart grid. In: 2013 International Conference on Connected Vehicles and Expo (ICCVE). IEEE, Las Vegas. pp 81-86

Haslak T (2020) Weighted fair queuing as a scheduling algorithm for deferrable loads in smart grids. In: Bertsch V, Ardone A, Suriyah M, Fichtner W, Leibfried T, Heuveline V (eds). Advances in Energy System Optimization. Springer, Cham. pp 123-141 
Hekkelman B, Poutré HL (2020) Fairness in power flow network congestion management with outer matching and principal notions of fair division. In: Proceedings of the Eleventh ACM International Conference on Future Energy Systems (e-Energy '20). Association for Computing Machinery, Virtual Event, Australia. pp 106-115

Hoßfeld T, Skorin-Kapov L, Heegaard PE, Varela M (2018) A new QoE fairness index for QoE management. Qual User Experience 3(1):4

Hu J, You S, Lind M, Østergaard J (2014) Coordinated charging of electric vehicles for congestion prevention in the distribution grid. IEEE Trans Smart Grid 5(2):703-711

IEA (2020) Global ev outlook 2020. Technical report, IEA. https://www.iea.org/reports/global-ev-outlook-2020

Islam MS, Mithulananthan N, Bhumkittipich K (2016) Feasibility of pv and battery energy storage based ev charging in different charging stations. In: 2016 13th International Conference on Electrical Engineering/Electronics, Computer, Telecommunications and Information Technology (ECTI-CON). IEEE, Chiang Mai. pp 1-6

Islam MS, Mithulananthan N, Lee KY (2018) Suitability of pv and battery storage in ev charging at business premises. IEEE Trans Power Syst 33(4):4382-4396

ITU (2008) ITU-T E.800 - SERIES E: Overall Network Operation, Telephone Service, Service Operation and Human Factors. https://www.itu.int/rec/T-REC-E.800-200809-I/en. Accessed 08 Mar 2021

Kong F, Liu X, Sun Z, Wang Q (2016) Smart Rate Control and Demand Balancing for Electric Vehicle Charging. In: 2016 ACM/IEEE 7th International Conference on Cyber-Physical Systems (ICCPS). IEEE, Vienna. pp 1-10

Lopes JAP, Soares FJ, Almeida PMR (2009) Identifying management procedures to deal with connection of electric vehicles in the grid. In: 2009 IEEE Bucharest PowerTech. pp 1-8

Martinenas S, Knezović K, Marinelli M (2017) Management of power quality issues in low voltage networks using electric vehicles: Experimental validation. IEEE Trans Power Delivery 32(2):971-979

Rezaei P, Frolik J, Hines PDH (2014) Packetized plug-in electric vehicle charge management. IEEE Trans Smart Grid 5(2):642-650

Rivera J, Goebel C, Jacobsen H-A (2015) A distributed anytime algorithm for real-time ev charging congestion control. In: Proceedings of the 2015 ACM Sixth International Conference on Future Energy Systems (e-Energy '15). ACM, New York. pp 67-76

Rudnik R, Wang C, Reyes-Chamorro L, Achara J, Boudec J-YL, Paolone M (2020) Real-time control of an electric vehicle charging station while tracking an aggregated power setpoint. IEEE Trans Ind Appl 56(5):5750-5761

Schlund J, Pruckner M, German R (2020) Flexability - modeling and maximizing the bidirectional flexibility availability of unidirectional charging of large pools of electric vehicles. In: Proceedings of the Eleventh ACM International Conference on Future Energy Systems (e-Energy '20). Association for Computing Machinery, New York. pp 121-132

Shi B, Liu J (2015) Decentralized control and fair load-shedding compensations to prevent cascading failures in a smart grid. Int J Electr Power Energy Syst 67:582-590

Ucer E, Kisacikoglu MC, Yuksel M, Gurbuz AC (2019) An internet-inspired proportional fair ev charging control method. IEEE Syst J 13(4):4292-4302

UI-Haq A, Buccella C, Cecati C, Khalid HA (2013) Smart charging infrastructure for electric vehicles. In: 2013 International Conference on Clean Electrical Power (ICCEP). IEEE, Alghero. pp 163-169

Wang B, Hu B, Qiu C, Chu P, Gadh R (2015) Ev charging algorithm implementation with user price preference. In: 2015 IEEE Power Energy Society Innovative Smart Grid Technologies Conference (ISGT). IEEE, Washington, DC. pp 1-5

Zenginis I, Vardakas JS, Zorba N, Verikoukis CV (2016) Analysis and quality of service evaluation of a fast charging station for electric vehicles. Energy 112:669-678

Zhou Y, Maxemchuk N, Qian X, Mohammed Y (2013) A weighted fair queuing algorithm for charging electric vehicles on a smart grid. In: 2013 IEEE Online Conference on Green Communications (OnlineGreenComm). IEEE, Piscataway. pp 132-136

Zhou Y, Maxemchuk N, Qian X, Wang C (2014) The fair distribution of power to electric vehicles: An alternative to pricing. In: 2014 IEEE International Conference on Smart Grid Communications (SmartGridComm). IEEE, Venice. pp 686-691

\section{Publisher's Note}

Springer Nature remains neutral with regard to jurisdictional claims in published maps and institutional affiliations.

\section{Submit your manuscript to a SpringerOpen ${ }^{\circ}$ journal and benefit from:}

- Convenient online submission

- Rigorous peer review

Open access: articles freely available online

- High visibility within the field

- Retaining the copyright to your article

Submit your next manuscript at $\gg$ springeropen.com 